\title{
SÍNDROME DE COGAN-REESE: RELATO DE UM CASO
}

\author{
COGAN-REESE SYNDROME: A CASE REPORT
}

Denise D.B. Silva'; Marcus A.G. de Matos ${ }^{1}$; Suryana A.R. Pessoa' ; Ana C. Ávila²; Hellmann Dantas²

\begin{abstract}
${ }^{1}$ Médicos, alunos do curso de especialização em Oftalmologia da Fundação Altino Ventura.
${ }^{2}$ Médicos Oftalmologistas. Departamento de Glaucoma da Fundação Altino Ventura.

Correspondência: Denise Dantas Boudoux Silva - Rua da Soledade, no. 136, Boa Vista - Recife, Pernambuco. CEP: $50.070-020$ Fone:

(0xx81) 421-4399 - Fax: (081) 421-4338 - E.mail: denisedb@hotlink.com.br
\end{abstract}

SILVA DDB; MATOS MAG; PESSOA SAR; ÁVILAAC \& DANTAS H. Síndrome de Cogan-Reese: relato de um caso. Medicina, Ribeirão Preto, 33: 82-87 jan./mar. 2000.

RESUMO: Modelo do estudo: Relato de caso

Importância do problema: A síndrome de Cogan Reese é uma rara patologia, classificada como uma das variantes da síndrome endotelial iridocorneana, que acomete indivíduos jovens, saudáveis, não correlacionada a história familiar, com evolução rápida de glaucoma de difícil controle.

Comentários: É descrito um caso de síndrome de Cogan Reese, epidemiologicamente atípico, assistido na Fundação Altino Ventura, Recife, Pernambuco, durante um período de seis meses. O paciente evolui com boa resposta à terapêutica clínica instituída, embora já apresentasse, ao diagnóstico, as conseqüências tardias de glaucoma secundário. São feitas considerações a respeito dos diversos aspectos da síndrome endotelial iridocorneana.

UNITERMOS: Doenças da Córnea. Endotélio da Córnea. Glaucoma. Doenças da Íris; síndrome; patologia.

\section{INTRODUÇÃO}

Normalmente, considera-se que a síndrome endotelial iridocorneana (ICE síndrome) tenha três variantes principais: síndrome de Chandler, atrofia progressiva (essencial) da íris e síndrome de Cogan Reese $^{(1 / 4)}$.

Predomina em leucodérmicos, do sexo feminino, com idade de apresentação, usualmente, a partir da terceira década de vida ${ }^{(1 / 3)}$. Casos familiares são $\operatorname{raros}^{(1,2,4)}$. É, freqüentemente, progressiva embora já tenha sido relatada a regressão de um caso ${ }^{(5)}$ e unilateral, embora alterações subclínicas contralaterais também possam ser observadas ocasionalmente ${ }^{(1 / 4,6)}$. Atribui-se sua etiopatogenia a uma inflamação endotelial crônica de etiologia provavelmente viral ${ }^{(2,3,6)}$.

Caracteriza-se pela presença de anormalidades corneanas características, com pleomorfismo e polimegatismo endotelial, semelhante a córnea goteada. Alterações distróficas irianas incluem atrofia, pseudopolicoria, ectrópio uveal. Os nódulos característicos da síndrome de Cogan Reese representam focos de estroma iriano, normal, remanescente, cercado por células endoteliais $^{(4)}$. É, freqüentemente, complicada com glaucoma ${ }^{(2 / 4)}$, provavelmente secundária ao crescimento da membrana corneana ectópica ${ }^{(2)}$. Sinéquias anteriores e periféricas ocorrem comumente ${ }^{(2,3)}$. Goniossinéquias múltiplas estendem-se em todas as direções, podendo bloquear o seio camerular. Pacientes com síndrome de Cogan-Reese queixam-se mais freqüentemente de alterações pupilares ${ }^{(2)}$.

Alguns estudos histológicos e à microscopia eletrônica ${ }^{(6 / 10)}$ demonstraram uma membrana de Descemet ectópica sobre a superfície corneana pos- 
terior e anterior da íris, ${ }^{(5,7 / 9)}$ a presença de necrose celular e de uma inflamação crônica, de leve intensidade, com perda de inibição de contato, associada à formação de múltiplas camadas endoteliais. Essas alterações podem, ou não, acometer todo o endotélio ${ }^{(11)}$. A microscopia especular mostra alterações endoteliais patognomônicas, com a presença de células anormais, caracterizadas por uma área escura com mancha central clara e, freqüentemente, uma zona periférica clara. O tamanho das células varia, mas, tipicamente, são maiores que as células endoteliais normais. Elas ocorrem em áreas que se apresentam com o aspecto de metal batido à reflexão especular no exame à lâmpada de fenda. São denominadas ICE-cells ${ }^{(4,12)}$.

\section{APRESENTAÇÃO DO CASO}

M.B.A, 43 anos, sexo masculino, melanodermo, pintor, natural e procedente de São Lourenço da Mata - PE, atendido na Fundação Altino Ventura, em setembro de 1998, queixando-se de diminuição progressiva da acuidade visual no olho direito, há alguns anos. Vinha encaminhado por oftalmologista de outro serviço, por apresentar pressão ocular elevada, tendo sido prescrito betaxolol colírio, uma gota de 12/12 h.
Vinha em uso regular da medicação. Negava antecedentes patológicos pessoais e familiares.

Ao exame oftalmológico, possuía acuidade visual para longe com correção $(-5,00 \mathrm{DE}<>-2,00 \mathrm{DC}$ a $90^{\circ}$ ) de 20/60 no olho direito (OD) e, no olho esquerdo (OE), (+ 0,50 DE) 20/20. À biomicroscopia, apresentava, no OD, córnea com aparência de metal batido difusamente, câmara anterior profunda (Grau IV de Van Herick) e opticamente livre, atrofia estromal iriana difusa, com nódulos pigmentados entre cinco e dez horas, pupila ovalada, com corectopia ínfero-temporal e reflexo fotomotor lentificado. Não foi observado ectrópio uveal (Figura 1). A pressão ocular (PO) era de $23 \mathrm{mmHg}$ no $\mathrm{OD}$ e $10 \mathrm{mmHg}$ no $\mathrm{OE}$, às 14 horas e 30 minutos. À gonioscopia, observou-se, em ambos os olhos, ângulo amplo (grau IV da classificação de Shaffer) em todos os quadrantes. No OD, observou-se área de hiperpigmentação, estendendo-se da raiz da íris ao trabeculado (às sete horas). Não observamos sinéquias periféricas. À fundoscopia, o OD apresentava nervo óptico pálido, escavação papilar total, atrofia de epitélio pigmentar da retina peripapilar, vasos de calibres e contornos normais, mácula sem alterações visíveis, retina aplicada (Figura 2). O olho esquerdo encontrava-se normal à avaliação.

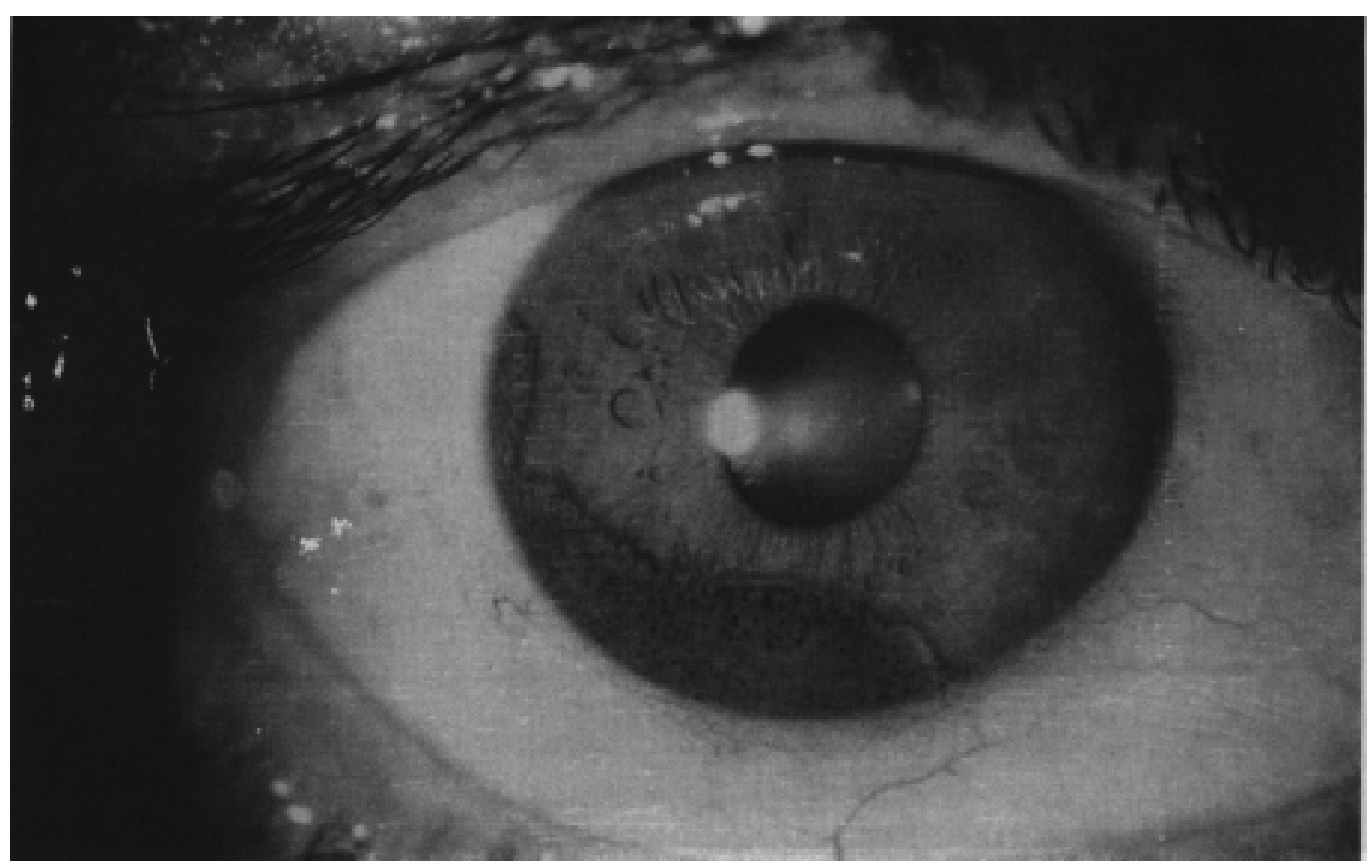

Figura 1: OD evidenciando atrofia estromal iriana difusa, com nódulos pigmentados, entre cinco e dez horas, corectopia infero-temporal. 
Considerou-se a hipótese diagnóstica de síndrome de Cogan-Reese no OD. Substituiu-se o betaxolol pelo maleato de timolol, e associou-se a dorzolamida de $12 / 12$ h, no OD.

Foi realizada microscopia especular (TOPCOM SP 100®) em ambos os olhos, o que evidenciou diminuição importante da contagem celular no OD, com alterações características que confirmavam o diagnóstico. (Figura 3). A perimetria computadorizada (Allergan Humphrey Field Analyser) demonstrou redução geral da sensibilidade no olho afetado. A análise de fibras nervosas (GDX laser Diagnosis Technology Incorporation), no OD, apresentava diminuição importante da espessura da camada de fibras nervosas nos pólos superior e inferior, com alteração da curva base que se retifica. (Figura 4). O olho esquerdo encontrava-se dentro dos padrões da normalidade.

O paciente foi acompanhado por seis meses, mensalmente, nesta instituição, Fundação Altino Ventura, apresentando, com as medicações prescritas, manutenção do quadro oftalmológico, com controle adequado da pressão ocular.

\section{DISCUSSÃO}

A síndrome endotelial iridocorneana é uma rara síndrome. Predomina em pessoas leucodérmicas, do

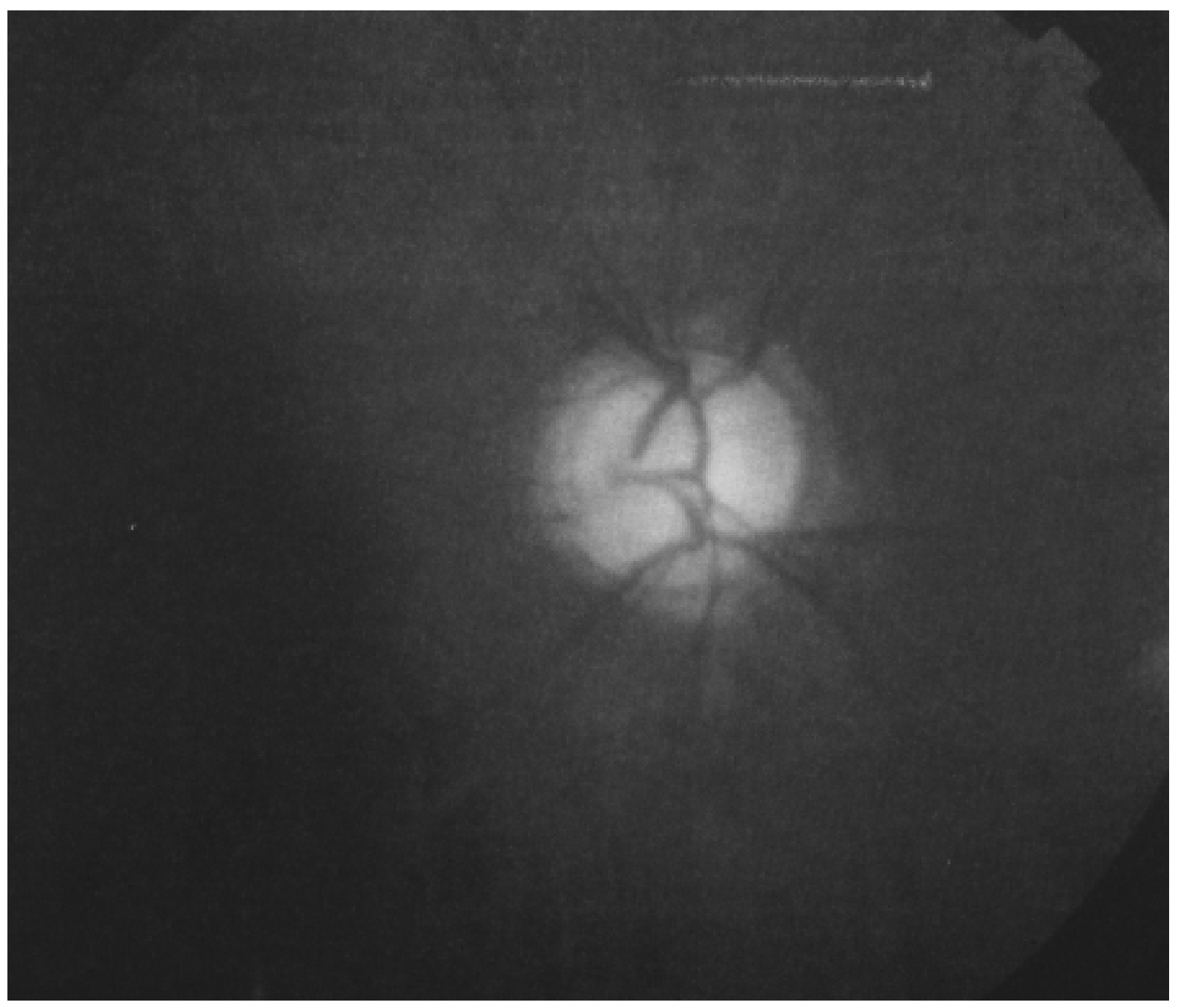

Figura 2: Retinografia do olho direito que mostra: escavação papilar total, atrofia de epitélio pigmentar da retina peripapilar, vasos de calibres e contornos normais, mácula sem alterações visíveis, retina aplicada. 


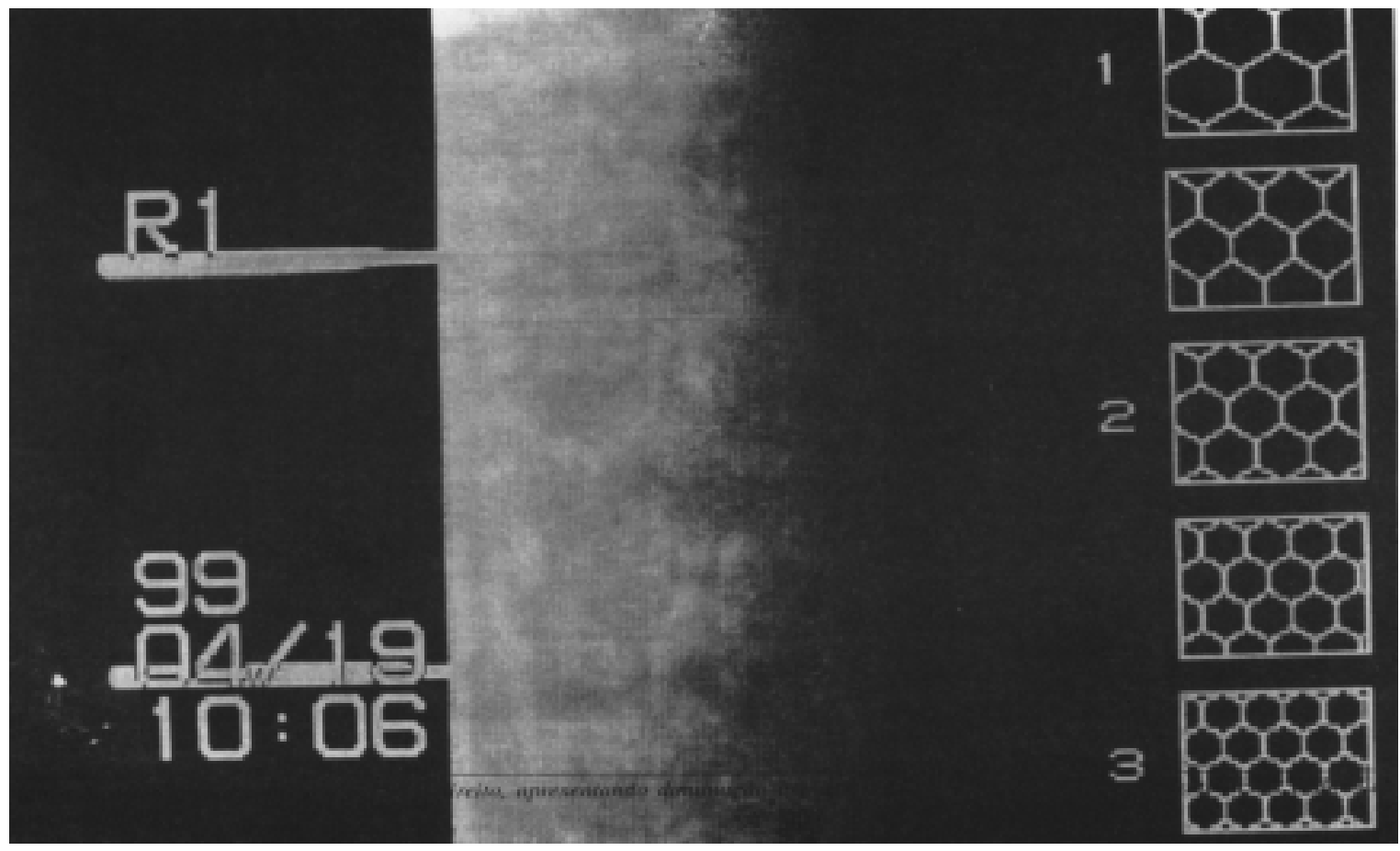

Figura 3: Microscopia especular do olho direito, apresentando diminuição importante do número de células, com alterações endoteliais caractrerísticas.

sexo feminino, com idade de apresentação, usualmente, a partir da terceira década de $\operatorname{vida}^{(1 / 3)}$. É, freqüentemente, progressiva e unilateral ${ }^{(1 / 3,4,6)}$. Há relatos de casos bilaterais, mais comuns em homens ${ }^{(2)}$. Numa série de Calixto e Cronemberger, observou-se que a apresentação inicial da síndrome de Cogan-Reese ocorria entre 30,3 +/- 11,6 anos e acometeu o sexo masculino em $21,4 \%$ dos casos $^{(2)}$.

Na Fundação Altino Ventura, diagnosticaramse quatro casos de ICE-síndrome, sendo este o único caso de síndrome de Cogan-Reese, diagnosticado na instituição. Há dois casos de atrofia essencial da íris e um caso de síndrome de Chandler, recentemente diagnosticado.

No caso relatado, observou-se comprometimento unilateral num indivíduo do sexo masculino com 43 anos. Não havia casos semelhantes na família ${ }^{(1 / 4)}$.

A síndrome de Cogan-Reese pode conter algum grau das características presentes nas outras variantes, porém, o seu principal achado são os nódulos na superfície da íris, circundados pela típica membrana celular ${ }^{(1 / 3,8)}$. O paciente observado apresentava córnea goteada, com áreas de atrofia estromal iriana e nódulos pigmentados, pediculados, corectopia e al- teração da forma pupilar (pupila ovalada),o que pode, tipicamente, caracterizar a patologia como síndrome de Cogan-Reese.

Acredita-se que o glaucoma seja causado por proliferação do endotélio corneano, com formação de membrana que, progressivamente, reveste a malha trabecular, e não devido às gonossinéquias que surgem tardiamente ${ }^{(2,3)}$.

O tratamento clínico do glaucoma deve ser tentado, inicialmente, com agentes que diminuam a produção de humor aquoso, embora muitos pacientes, eventualmente, necessitem cirurgia filtrante. Vários procedimentos cirúrgicos filtrantes têm sido relatados na literatura, com sucesso variado no controle da pressão ocular ${ }^{(2,3,13)}$. Nos casos rebeldes de edema corneano, a ceratoplastia penetrante pode ser necessária após controle tensional ${ }^{(2,3)}$. O paciente encontra-se com boa resposta ao tratamento clínico instituído, como é comum em alguns casos, particularmente no início do quadro $^{(1 / 3)}$.

\section{Agradecimento}

À professora Sylvia Lemos Hinrichsen, pela orientação metodológica. 

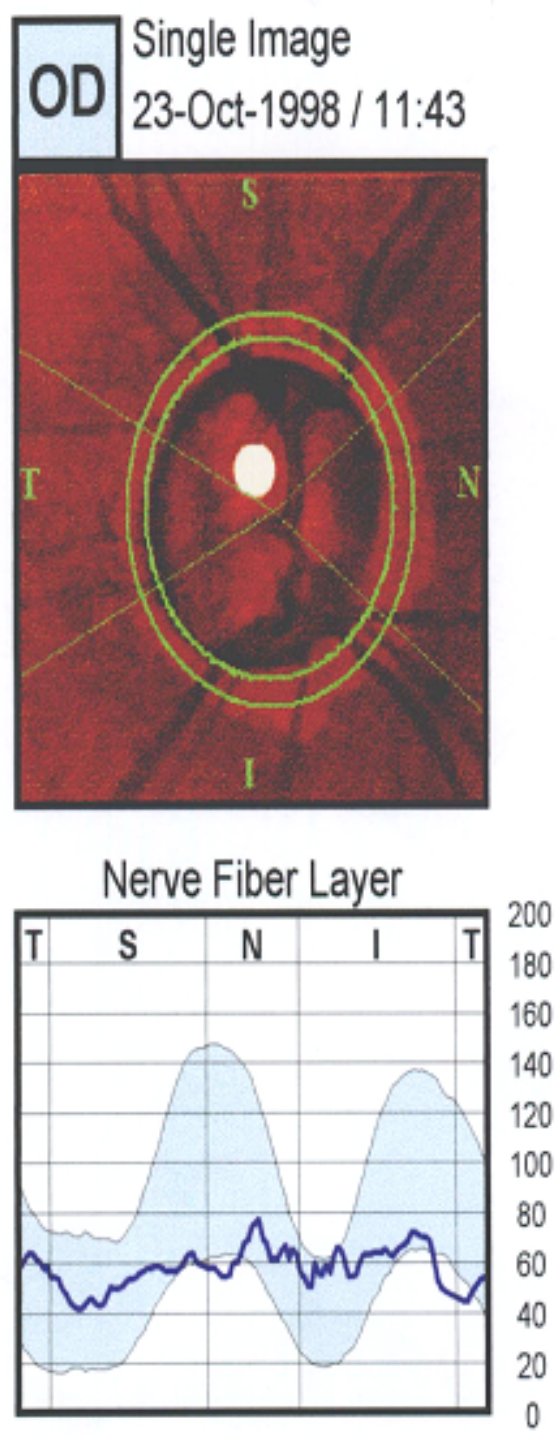
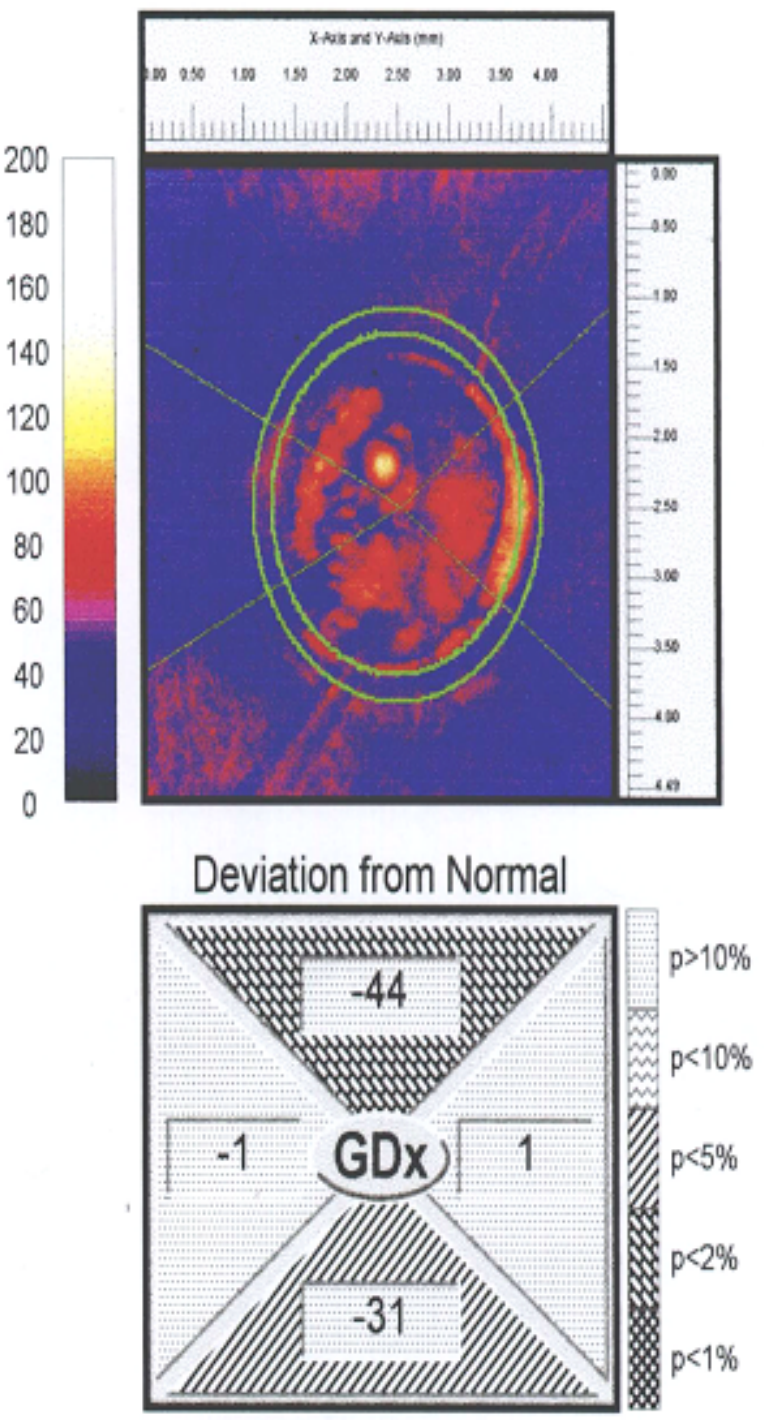

NERVE FIBER ANALYSIS

\begin{tabular}{|c|c|c|c|c|c|c|c|}
\hline & Act. Value & Status & Probabelity & & Act. Value & Status & Probability \\
\hline Symmetry & 0.89 & Within Normal & & The Number & 72 & & \\
\hline Superior Ratio & 1.26 & Outside Normal & $2.9 \%$ & Average Thickness & 55 & Within Normal & \\
\hline Inferior Ratio & 1.41 & Borderline & $6.1 \%$ & Ellipse Average & 59 & Within Normal & \\
\hline SuperioriNasal & 1.21 & Outside Normal & $0.2 \%$ & Superior Average & 54 & Outside Normal & $3.0 \%$ \\
\hline Nax. Modulation & 0.41 & Outside Normal & $0.5 \%$ & Inferior Average & 61 & Borderline & $8.2 \%$ \\
\hline Ellipse Modulation & 0.91 & Outside Normal & $0.9 \%$ & Superior Integral & 0.139 & Outside Normal & $2.6 \%$ \\
\hline
\end{tabular}

Figura 4: Análise de fibras nervosas do olho direito. Note-se a diminuição de espessura, nas camadas de fibras nervosas, superior e inferiormente. 
SILVA DDB; MATOS MAG; PESSOA SAR; ÁVILA AC \& DANTAS H. Cogan-Reese syndrome: a case report. Medicina, Ribeirão Preto, 33: 82-87 jan./march 2000.

ABSTRACT: Paper design: Case Report

Clinical relevance: Cogan-Reese syndrome is an uncomun disease, classified as one of the three kinds of iridocorneal endothelial syndrome, affects young and healthy people, without inherance pattern, that quickly evolves to a difficult controlling glaucoma.

Comments: A Cogan-Reese syndrome case, assisted at Altino Ventura Foundation, Recife, Pernambuco, during a period of six months, is described. The outcome was successful with clinical treatment, thought associated to late features of secundary glaucoma on diagnosis. Comments on several aspects of iridocorneal endothelial syndrome are described.

UNITERMS: Corneal Diseases. Endothelium, Corneal. Glaucoma. Iris Diseases; pathology.; syndrome.

\section{REFERÊNCIAS BIBLIOGRÁFICAS}

1 - WILSON MC \& SHIELDS MB. A comparison of the clinical variations of the iridocorneal endothelial syndrome. Arch Ophthalmol 107: 1465-1468, 1989.

2 - CALIXTO N \& CRONEMBERGER S. Glaucoma e distrofias endoteliais corneanas. In: ALMEIDA HG; ALMEIDA GV; CALIXTO N \& CARVALHO CA. Glaucomas secundários, Roca, São Paulo, p. 291-298, 1985

3 - SHIELDS MB. Glaucoma associado a alterações primárias do endotélio corneano. In:SHIELDS MB. Glaucoma, $2^{a}$ ed, Panamericana, São Paulo, p. 235-245, 1989.

4 - LAGANOWISKI HC; SHERRARD ES; MUIR MG \& BUCKLEY RJ. Distinguishing features of the iridocorneal endothelial syndrome and posterior polymorphous dystrofy: value of endothelial specular microscopy. Br J Ophthalmol 75: 212216, 1991.

5 - BOURNE WM \& BRUBACKER RF. Progression and regression of partial corneal involvement in the iridocorneal endothelial syndrome. Trans Am Ophthalmol Soc 90: 201224,1992

6 - HUNA R; BARAK A \& MELAMED S. Bilateral iridocorneal endothelial syndrome presented as Cogan-Reese and Chandler's syndrome. J Glaucoma 5: 60-62, 1996.

7 - ALVARADO JA; MURPHY CG; MAGLIO M \& HETHERINGTON J. Pathogenesis of Chandler's syndrome, essential iris atrophy and the Cogan Reese syndrome. II. Estimated age at disease onset. Invest Ophtalmol Vis Sci 27: 873-882, 1986
8 - RADIUS RL \& HERSCHLER J. Histopathology in the irisnevus (Cogan-Reese) syndrome. Am J Ophthalmolol 89: 780-786, 1980.

9 - EAGLE RC JR; FONT RL; YANOFF M \& FINE BS. The iris naevus (Cogan Reese) syndrome: light and electron microscopic observations. Br J Ophhtalmol 64: 446-452, 1980.

10 - KHALIL MK \& FINLAYSON MH. Electron microscopy in iris nevus syndrome. Can J Ophthalmol 15: 44-48, 1980.

11 - ALVARADO JA; MURPHY CG; MAGLIO M \& HETHERINGTON J. Pathogenesis of Chandler's syndrome, essential iris atrophy and the Cogan Reese syndrome. I. Alterations of the corneal endothelium. Invest Ophtalmol Vis Sci 27: 853-872, 1986.

12 - HIRST LW; QUIGLEY HA; STARK WJ \& SHIELDS NB. Specular microscopy of iridocorneal-endothelial syndrome. Aust J Ophthalmol 8: 139-146, 1980.

13 - KIDD M; HETHERINGTON J \& MAGEE S. Surgical results in Iridocorneal Endothelial Syndrome. Arch Ophthalmo 106: 199-201, 1988

Recebido para publicação em 13/10/1999

Aprovado para publicação em 03/03/2000 